\title{
Research on the Output Characteristics of Microfluidic Inductive Sensor
}

\author{
Xingming Zhang, Hongpeng Zhang, Yuqing Sun, Haiquan Chen, and Yindong Zhang \\ Department of Marine Engineering, Dalian Maritime University, Dalian, China \\ Correspondence should be addressed to Hongpeng Zhang; zhppeter@163.com
}

Received 15 October 2013; Revised 17 January 2014; Accepted 17 January 2014; Published 13 March 2014

Academic Editor: Guillaume Tresset

Copyright (C) 2014 Xingming Zhang et al. This is an open access article distributed under the Creative Commons Attribution License, which permits unrestricted use, distribution, and reproduction in any medium, provided the original work is properly cited.

\begin{abstract}
This paper focuses on the output characteristic of the microfluidic inductive sensor. First the coil-metal particle system model is established from Maxwell's equations. Then series solution is achieved by solving partial differential equation. Finally the numerical simulations and physical experiments are compared on particle feature, particle size, excitation frequency, coil turns, and coil density. The experiments coincide well with the simulations.
\end{abstract}

\section{Introduction}

The development of high-precision hydraulic technology in recent years has resulted in increased hydraulic oil purity requirement. When abnormal wear occurs, particle concentration gradually increases and the size grows from about $50 \mu \mathrm{m}$ to $100 \mu \mathrm{m}$ [1]. An effective method of preventing hydraulic system failure is to carry out the oil abrasive detection work, thus keeping the degree of contamination within the tolerance range of key components [2].

The current techniques to monitor particle pollution in hydraulic oil include iron spectrum measurement analysis, pollution gravimetric method, microscope comparison method, and particle counting method. Except for particle counting, all these methods can only estimate the pollution to some extent, and the results are greatly influenced by human factors. Particle counting is a common method of detecting fluid contamination, which can determine the size distribution of oil contaminants by measuring the number and amplitude of signal pulses caused by the particles [3]. Research on particle counting has focused on the optical detection, acoustic detection, capacitive sensing, and the inductance detection methods. A list of these oil particle detection methods is featured in Table 1. As for the other methods, iron spectrum measurement analysis and pollution gravimetric method cannot give the particle size distribution, whereas the microscope comparison and particle counting methods excluded inductance method which cannot distinguish ferromagnetic or nonferromagnetic metal particles.

Du et al. $[4,5]$ have done series of works on microfluidic inductive sensors [5]. The authors also have proposed some previous works to apply a microfluidic technique to detect wear debris in hydraulic oil using inductive sensing [6]. There we examined the correlation between the coil line width and inductance variation [6]. The amount of pulse generated by copper or iron particles indicates the number of particles, whereas the height indicates their sizes. Because the relative permeability of nonferromagnetic metal particles is very close to 1 , the magnetization can be ignored in static magnetic field or low frequency harmonic magnetic field. Therefore DC (direct current) or low frequency methodologies fail. Eddy current within nonferromagnetic metal particle produced by medium or high frequency excitation decreases average relative permeability, which produces demagnetization effect. So both ferromagnetic and nonferromagnetic metal particles could be detected by alternating current (AC) inductance method. The current work examines the basic principles and output characteristics of this method by undertaking research using the complex Helmholtz equation established from the Maxwell equations. Then, the correlation between various parameters and inductance variation is obtained from 
TABLE 1: Listing of oil particle detection methods.

\begin{tabular}{llll}
\hline Method & Working principle & Features & Ref. \\
\hline Optical & Light blocking or light scattering & Sensitive but easy to be interfered by air bubbles and oil oxidation & [11-13] \\
Acoustic & Reflection of sound waves & Easy to be interfered by background noises and temperature \\
Capacitive & Dielectric difference & Sensitive but easy to be interfered by water content and acidity \\
Inductive & Magnetic flux change & Able to distinguish metal particles & {$[16,15]$} \\
\hline
\end{tabular}

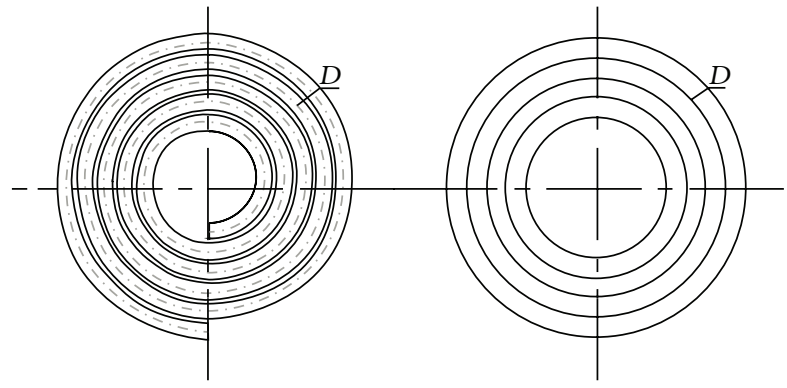

FIgURE 1: Approximation of the spiral planar coil.

a series solution of a chip model, which is confirmed by the experiments.

\section{Model Establishment and Series Solution of the Coil-Metal Particle System}

This process is completed based on several factors. Firstly, the hysteresis in the particle is ignored, and the conductivity and dielectric constant are assumed to be constant. Secondly, for the substrate of the chip and the polydimethylsiloxane (PDMS), the conductivity and relative permeability are assumed to be 0 and 1, respectively. Finally, the planar coil is assumed to be a series of concentric circles, as shown in Figure 1. The final assumption is based on the fact that the spiral planar coil in the microfluidic chip is thin and narrow enough.

When a metal particle passes radially above the spiral planar coil, peak inductance change appears in the metal particle located at the axis of the coil in a spherical coordinate system $(R, \Theta, \Phi)$ as shown in Figure 2 . In that system, the center $O$ of the spherical particle with radius $a$ is set to the coordinate origin, and the $Z$ axis passes through the center $O^{\prime}$ of the $M$ turns of planar coil. The distance between the center of the particles and the center of the planar coil is represented by $z_{0}$. The $m$ th $(1 \leq m \leq M)$ current ring in spherical coordinate is expressed as $\left(r_{m}, \theta_{m}, \varphi\right) ; r_{m}$ is distance between center of spherical particle and current element on the $m$ th current ring; its radius is given by $\rho_{m}=\sqrt{r_{m}^{2}+z_{0}^{2}}$, which is excited by $\dot{I}=I e^{j \omega t}$.

The magnetic vector potential $\dot{A}=e^{j \omega t} \vec{A}(r, \theta, \phi)$ at any point can be obtained using the complex Helmholtz equation [7], which can be derived from a differential form of Maxwell's field equation [8] given by the following:

$$
\nabla^{2} \vec{A}+k^{2} \vec{A}=0
$$

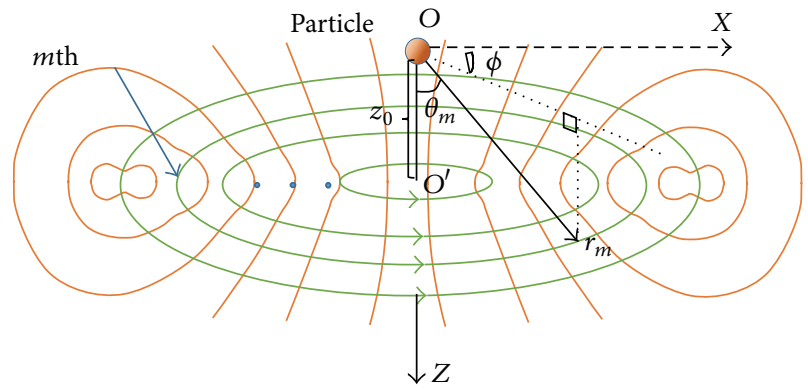

$$
\begin{aligned}
& \longrightarrow \text { Magnetic induction line } \\
& >\quad \text { Current }
\end{aligned}
$$

Figure 2: The model for the coil-metal particle system. In the magnetic field produced by planar coil, the metal particle is magnetized.

where $\omega$ is the angular velocity of the excitation alternating current; $\mu_{r}$ is the relative permeability, which is 1000 for iron and 0.99991 for copper; $\mu_{0}$ is the permeability of the vacuum; $\varepsilon_{0}$ is the permittivity of the vacuum; $\varepsilon_{r}$ is the relative permittivity, which is 1 for both iron and copper; $\sigma$ is the conductivity of particles, whose value is $5.8 \times 10^{7} \mathrm{~S} / \mathrm{m}$ for copper and $1.0 \times 10^{7} \mathrm{~S} / \mathrm{m}$ for iron; and $j$ is the imaginary unit. $k$ is the wave number $k^{2}=\omega^{2} \mu_{r} \mu_{0} \varepsilon_{0}\left(\varepsilon_{r}-j\left(\sigma / \varepsilon_{0} \omega\right)\right)$, which can be ignored outside the metal particles. Then partial differential equation of magnetic vector potential distribution outside the particle is given by

$$
\nabla^{2} \vec{A}=0
$$

which is the harmonic equation.

From the series solutions [9] for the Helmholtz equation (1) and the harmonic equation (2), the magnetic vector potential with any current ring in the spherical coordinates can be derived through the continuity of the magnetic vector potential and the tangential components of the magnetic field on the particle surface [10]. Consider

$$
A_{\varphi}(r, \theta)=\left\{\begin{array}{c}
\frac{\mu_{0} I}{2} \sum_{n=0}^{\infty} \frac{(2 n+1) a^{n} R_{n}\left(r_{m}\right)}{f_{n}(a) n(n+1)} j_{n}(k r) P_{n}^{1}(\cos \theta), \\
r \leq a, \\
\frac{\mu_{0} I}{2} \sum_{n=0}^{\infty} \frac{R_{n}\left(r_{m}\right)}{n(n+1)}\left[r^{n}+\frac{g_{n}(a)}{r^{n+1}}\right] P_{n}^{1}(\cos \theta), \\
a<r \leq r_{m}, \\
\frac{\mu_{0} I}{2} \sum_{n=0}^{\infty} \frac{R_{n}\left(r_{m}\right)}{n(n+1)}\left[r_{m}^{2 n+1}+g_{n}(a)\right] \frac{P_{n}^{1}(\cos \theta)}{r^{n+1}}, \\
r>r_{m}
\end{array},\right.
$$


In the equation above, $P_{n}^{1}(\cdot)$ is the $n$ degree and 1 order of the associated Legendre function. Meanwhile, $g_{n}(a)$ and $f_{n}(a)$ are defined in (4) and (5), respectively. Both functions depend on the magnetic and electrical properties as well as on the size of the particles. Consider the following:

$$
\begin{gathered}
g_{n}(a)=\frac{\left(n \mu_{r}+\mu_{r}+n\right) j_{n}(k a)+k a j_{n-1}(k a)}{n\left(\mu_{r}-1\right) j_{n}(k a)-k a j_{n-1}(k a)} a^{2 n+1}, \\
f_{n}(a)=\frac{k a j_{n-1}(k a)+n\left(\mu_{r}-1\right) j_{n}(k a)}{\mu_{r}(2 n+1)} .
\end{gathered}
$$

In addition, $j_{n}(\cdot)$ is the $n$ order of first kind spherical Bessel function. $R_{n}\left(r_{m}\right)$ depends on the coordinate of the current ring and is defined as follows:

$$
R_{n}\left(r_{m}\right)=\frac{\rho_{m}}{r_{m}^{n+1}} P_{n}^{1}\left(\frac{z_{0}}{r_{m}}\right) .
$$

The magnetic vector potential scattered by particle is named $A_{\mathrm{sc}}$. The $A_{\mathrm{sc}}$ field changes the magnetic flux in the current ring, from which inductance and mutual inductance can be derived. The $A_{\text {sc }}$ field outside of the particles can be obtained by

$$
A_{\mathrm{sc}}=A-\left.A\right|_{a \rightarrow 0}=\frac{\mu_{0} I}{2} \sum_{n=0}^{\infty} \frac{R_{n}\left(r_{m}\right) g_{n}(a)}{n(n+1) r^{n+1}} P_{n}^{1}(\cos \theta) .
$$

For any two current rings $m 1$ and $m 2$, the $A_{\text {sc }}$ field impact on the $m 2$ th current ring caused by the $m 1$ th current ring is given by

$$
\begin{array}{r}
A_{\mathrm{sc}}\left(r_{m 1}, r_{m 2}\right)=\frac{\mu_{0} I}{2} \sum_{n=0}^{\infty} \frac{R_{n}\left(r_{m 1}\right) g_{n}(a)}{n(n+1) r_{m 2}^{n+1}} P_{n}^{1}\left(\cos \theta_{m 2}\right), \\
m 1, m 2 \in\{1,2,3, \ldots, M\} .
\end{array}
$$

Then, the change of the magnetic flux caused by $A_{\mathrm{sc}}$ can be obtained by applying the second kind of curvilinear integral on the current ring using

$$
\Delta \Phi_{m}=\int_{l} \vec{A}_{s c} d \vec{l},
$$

where $l$ is the current path. From (9), the change of inductance and mutual inductance between any two current rings can be derived by

$$
\Delta L_{m 1, m 2}=\frac{2 \pi \rho_{m 2} A_{\mathrm{sc}}\left(r_{m 1}, r_{m 2}\right)}{I} .
$$

The total inductance of the $M$-turn planar coil is derived by summing up all the impedance changes using the equation given by

$$
\Delta L=\sum_{m 2=1}^{M} \sum_{m 1=1}^{M} \Delta L_{m 1, m 2}=\pi \mu_{0} \sum_{n=1}^{\infty} \frac{g_{n}(a) h_{n}}{n(n+1)},
$$

where $h_{n}$ defined in (12) depends on the degree of planar coil density; its value also increases with the increasing
TABLE 2: Chips' specification in the experiment.

\begin{tabular}{lccc}
\hline Number & Turns & Line width/gap width & Outer diameter \\
\hline $\mathrm{C}_{1}$ & 35 & $74 \mu \mathrm{m} / 74 \mu \mathrm{m}$ & $10.64 \mathrm{~mm}$ \\
$\mathrm{C}_{2}$ & 30 & $74 \mu \mathrm{m} / 74 \mu \mathrm{m}$ & $9.16 \mathrm{~mm}$ \\
$\mathrm{C}_{3}$ & 25 & $74 \mu \mathrm{m} / 74 \mu \mathrm{m}$ & $7.68 \mathrm{~mm}$ \\
$\mathrm{C}_{4}$ & 20 & $74 \mu \mathrm{m} / 74 \mu \mathrm{m}$ & $6.20 \mathrm{~mm}$ \\
$\mathrm{C}_{5}$ & 30 & $74 \mu \mathrm{m} / 99 \mu \mathrm{m}$ & $10.64 \mathrm{~mm}$ \\
$\mathrm{C}_{6}$ & 25 & $74 \mu \mathrm{m} / 133 \mu \mathrm{m}$ & $10.64 \mathrm{~mm}$ \\
$\mathrm{C}_{7}$ & 20 & $74 \mu \mathrm{m} / 185 \mu \mathrm{m}$ & $10.64 \mathrm{~mm}$ \\
$\mathrm{C}_{8}$ & 15 & $74 \mu \mathrm{m} / 271 \mu \mathrm{m}$ & $10.64 \mathrm{~mm}$ \\
$\mathrm{C}_{9}$ & 10 & $74 \mu \mathrm{m} / 444 \mu \mathrm{m}$ & $10.64 \mathrm{~mm}$ \\
\hline
\end{tabular}

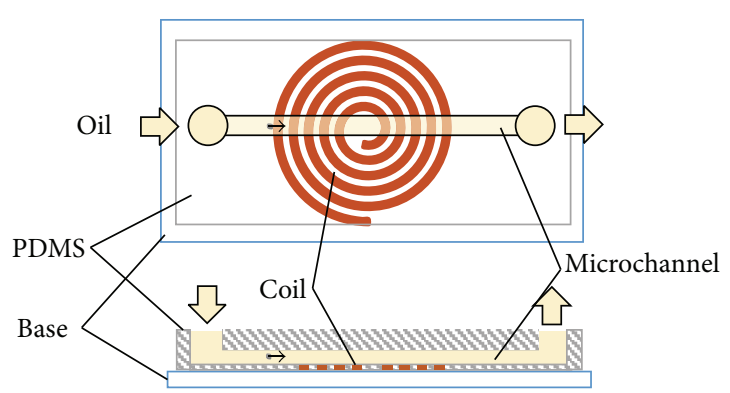

FiguRe 3: Schematic diagram.

degree of planar coil density. The real component of complex inductance $\Delta L$ changes the inductance of the planar coil, and the imaginary component influences its resistance. Consider

$$
h_{n}=\left[\sum_{m=1}^{M} R_{n}\left(r_{m}\right)\right]^{2} \text {. }
$$

\section{Experimental Verification}

3.1. Device Setup and Test Procedure. The performance of the planar metal particle counter is shown in Figure 3, which was demonstrated using a microfluidic device fabricated with PDMS microchannel and bonded to a glass substrate in conjunction with a planar inductor coil. Similar to the planar inductor, the microfluidic channel was also fabricated by soft lithography. Fabrication method was described in prior work [6]. The single channel sensor consisted of an inlet reservoir, an outlet reservoir, and a single channel with $600 \mu \mathrm{m}$ (height) $\times 600 \mu \mathrm{m}$ (width) $\times 30 \mathrm{~mm}$ (length) .

Nine different chips were fabricated with the parameters shown in Table 2. The planar coils from $\mathrm{C}_{1}$ to $\mathrm{C}_{4}$ were of the same line widths and gaps in order to research the impacts of radius position (Figure 5), coil turns (Figures 6 and 7), and excitation frequency (Figure 8) on inductance change. Furthermore, the chips in specifications from $\mathrm{C}_{5}$ to $\mathrm{C}_{9}$ with the same line widths and external diameters, but with different gaps and turns, were used to obtain the correlation between planar coil density and output (Figure 9).

The principle of signal processing system is shown in Figure 4. A $2 \mathrm{~V}$ AC voltage was applied between the planar 


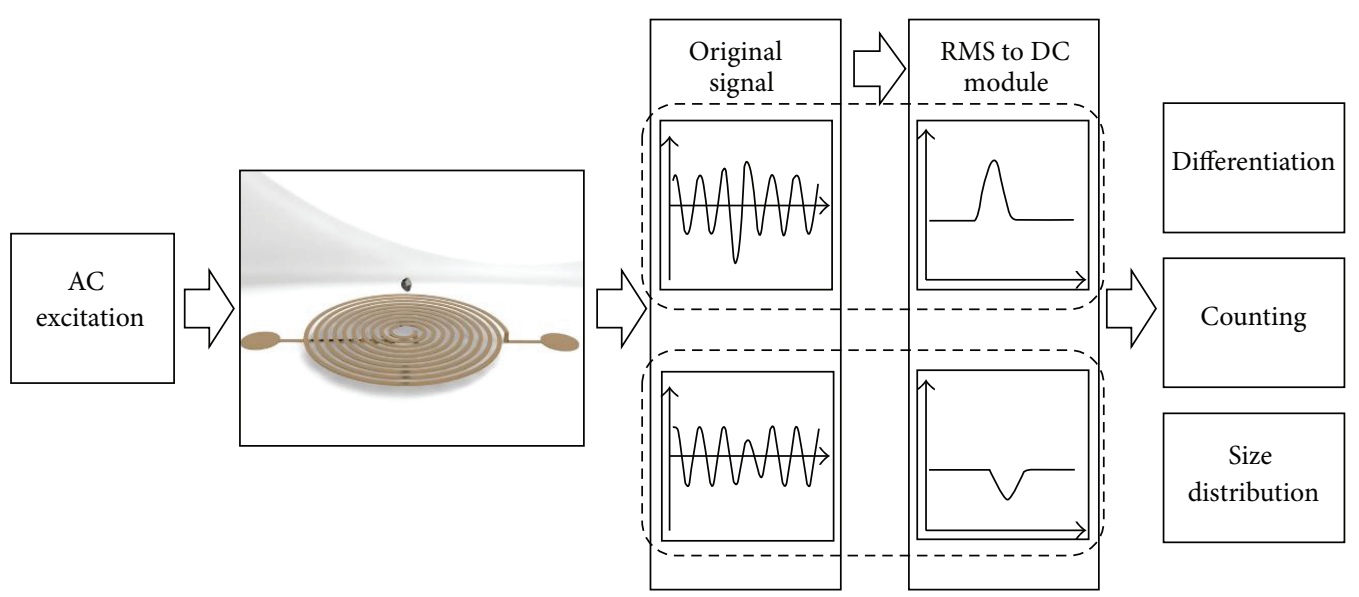

FIGURE 4: Working principle.

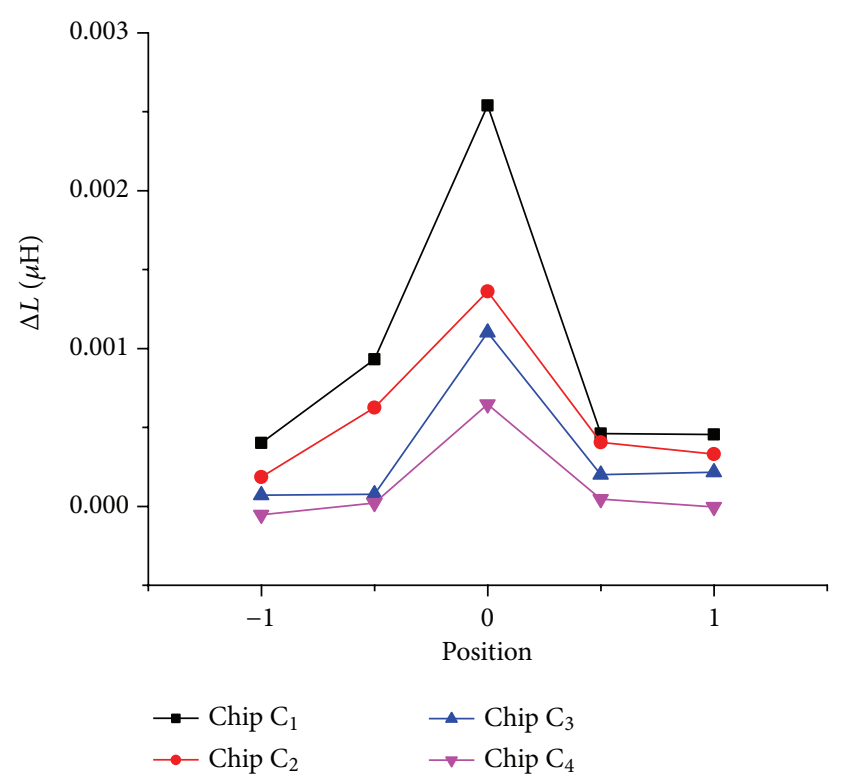

(a)

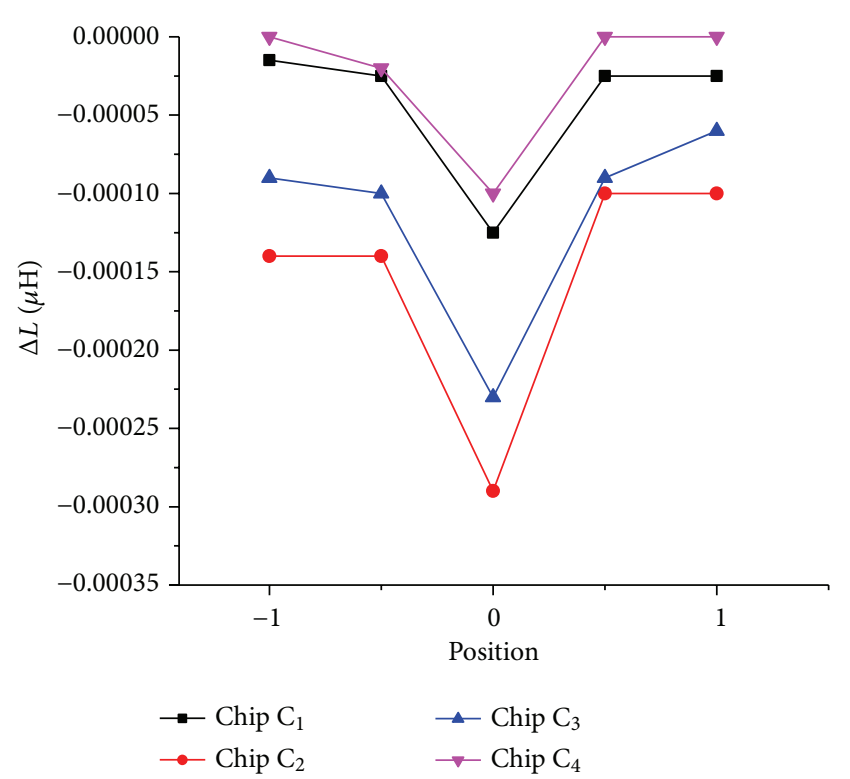

(b)

FIGURE 5: Inductance changes for a metal particle passing the center of the planar coils. (a) Iron and (b) copper.

coil and a constant impedance, which induced a harmonic magnetic field around the planar inductor coil. The AC was supplied by an LCR meter (Agilent E4980A). In the experimental setup, the voltage on the planar coil is amplified by an AD797 (Analog Devices, USA) and converted to DC by an AD637 (Analog Devices, USA). Then it was fed to the digital data acquisition system (NI USB 6259), which was used as a source-measure unit to measure the inductance changes of the planar coil.

Before the test, some iron and copper particles were selected with a microscope (NIKON Ti, Japan). The equivalent diameter was also measured by NIS-Element D (Nikon, Japan). Then these particles were carefully picked up by needle and transferred to a centrifuge tube. During the test, particles mixed with hydraulic oil (Hyspin AWH-M, Castrol) were transferred with a pipette to the inlet of microchannel.
The movement of particle is controlled by a syringe pump (Harvard).

3.2. Simulations and Contrast Experiments in the Harmonic Field. Figure 5 shows the inductance changes for a metal particle passing the center of the planar coils. In this test, an iron particle $(370 \mu \mathrm{m})$ and a copper particle $(340 \mu \mathrm{m})$ were selected and transferred inside the chips $\left(\mathrm{C}_{1}, \mathrm{C}_{2}, \mathrm{C}_{3}\right.$, and $\mathrm{C}_{4}$, resp.; 0 is the center of coil; -1 is the coil edge near inlet; 1 is the coil edge near outlet). The excitation frequency was $2 \mathrm{MHz}$. From the figures, one can see that iron particle increases the inductance of coil while copper particle decreases it. The sensitive area of the chip is located in the middle area of planar coil and the inductance peak appeared at center of planar coil, and that is due to outer current rings which 


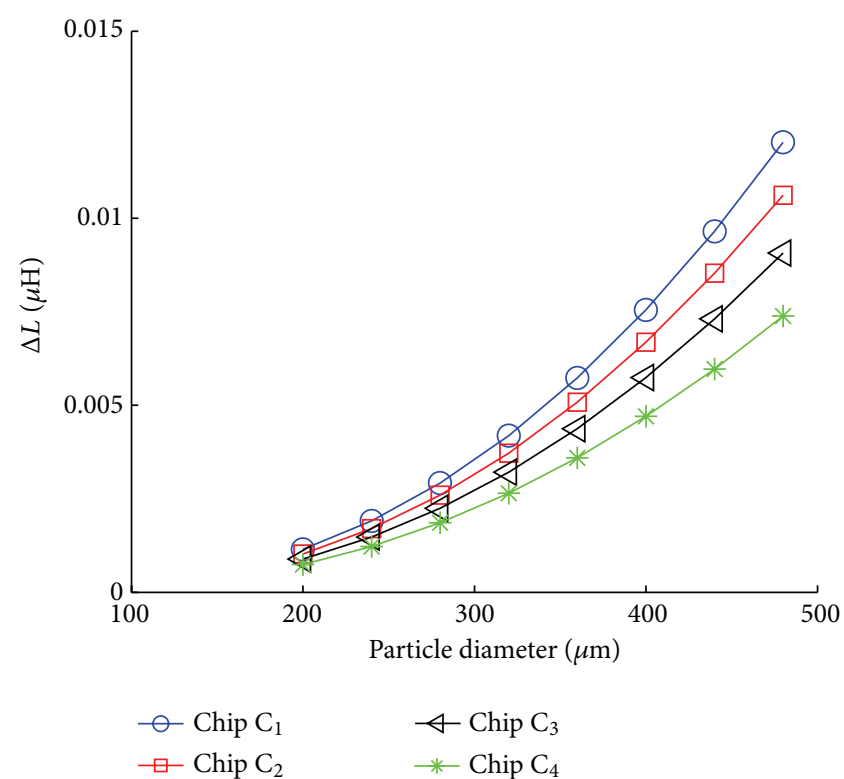

(a)

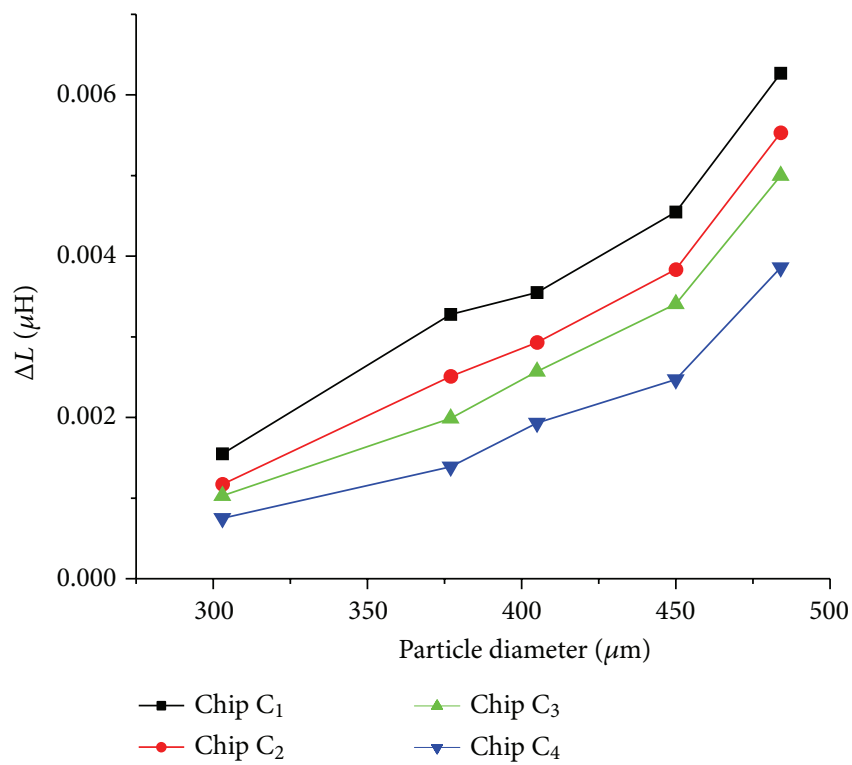

(b)

Figure 6: Inductance changes of planar coils $\left(\mathrm{C}_{1}, \mathrm{C}_{2}, \mathrm{C}_{3}\right.$, and $\left.\mathrm{C}_{4}\right)$ by different size iron particles. (a) Numerical simulations. (b) Experimental results.

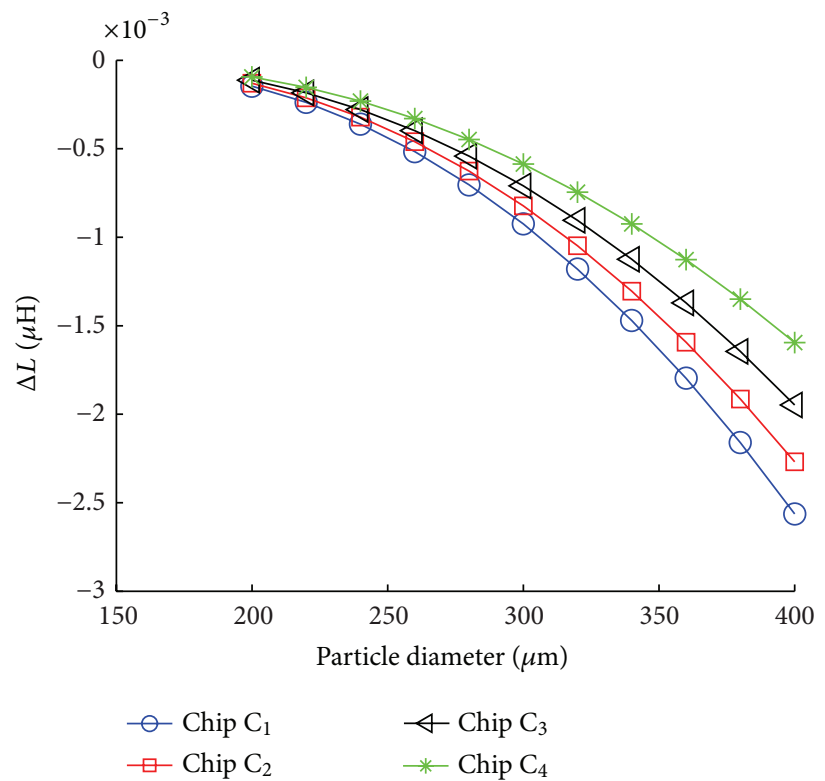

(a)

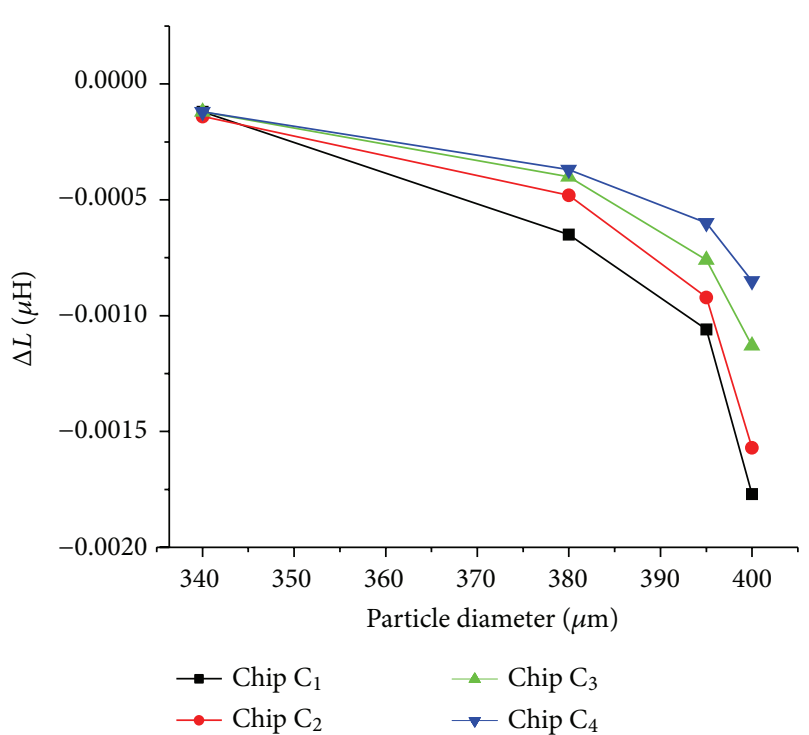

(b)

Figure 7: Inductance changes of planar coils $\left(\mathrm{C}_{1}, \mathrm{C}_{2}, \mathrm{C}_{3}\right.$, and $\left.\mathrm{C}_{4}\right)$ by different size copper particles. (a) Numerical simulations. (b) Experimental results.

are insensitive to particle. While the contribution of outer ring has less contribution to inductance change, in the same density, increasing the number of turns helps monitoring smaller particles.

Figure 6 is the inductance changes of planar coils $\left(\mathrm{C}_{1}\right.$, $\mathrm{C}_{2}, \mathrm{C}_{3}$, and $\mathrm{C}_{4}$ ) by iron particles, which are of the same coil density but of different coil turns. The excitation frequency is $2 \mathrm{MHz}$. The curve is upward slope with particle diameter increase. It is easy to understand that the bigger iron particle causes the larger inductance change. The reason is that the iron particle strengthens the magnetic flux of the coil, which therefore increases the coil inductance [5]. Furthermore, the more turns of coil contribute to the larger inductance change.

Figure 7 shows the inductance changes of planar coils $\left(\mathrm{C}_{1}\right.$, $\mathrm{C}_{2}, \mathrm{C}_{3}$, and $\mathrm{C}_{4}$ ) by copper particles, which are of the same coil density but of different coil turns. The curve is downward 


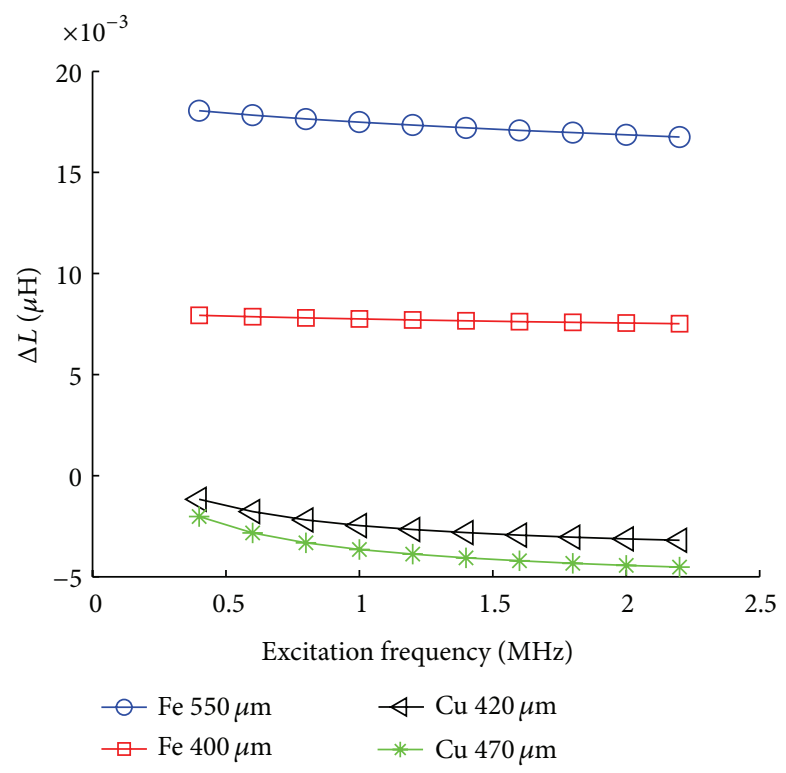

(a)

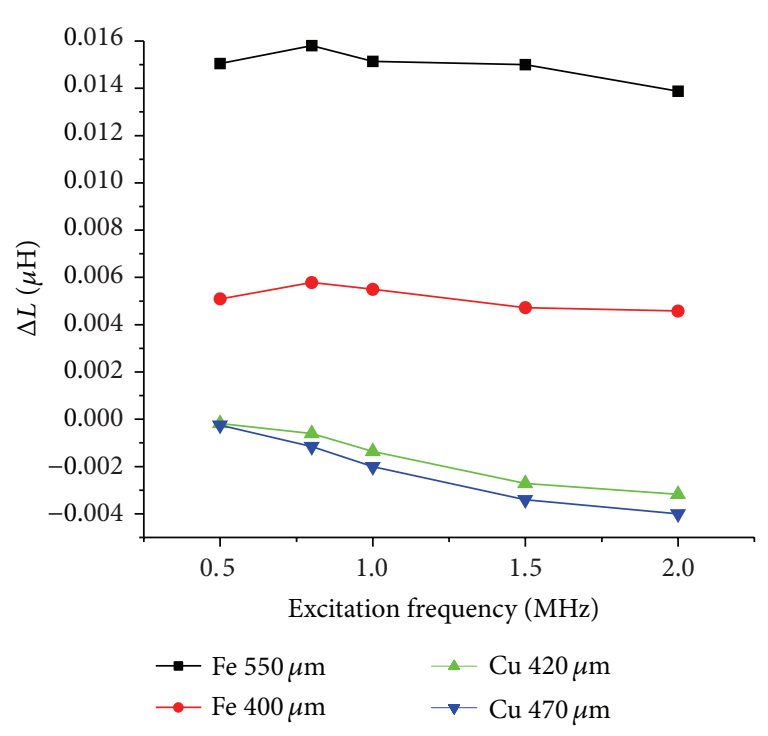

(b)

Figure 8: Inductance changes of the planar coil $\mathrm{C}_{1}$ with different excitation $(0.5-2.0 \mathrm{MHz})$. (a) Numerical simulations. (b) Experimental results.

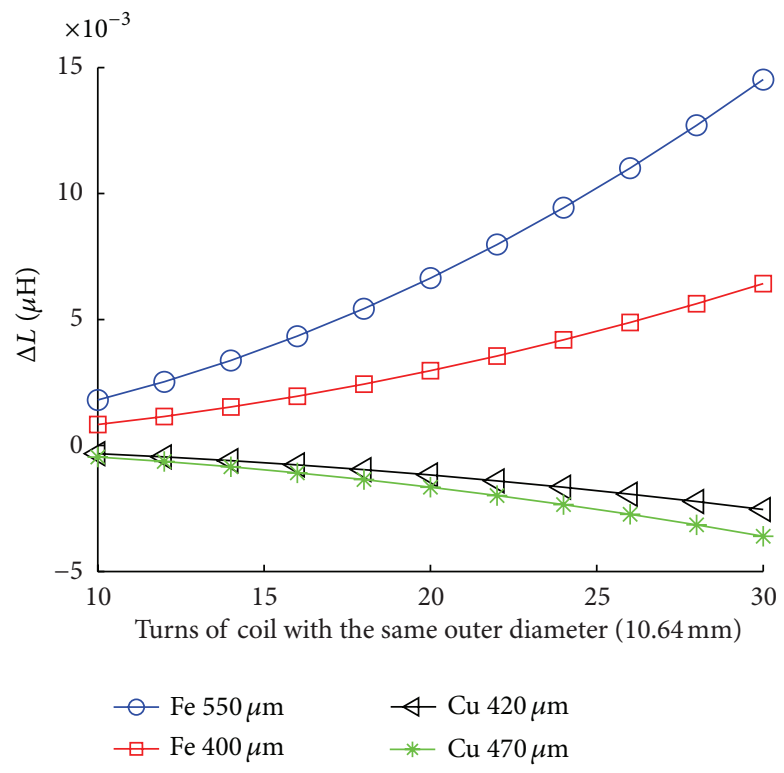

(a)

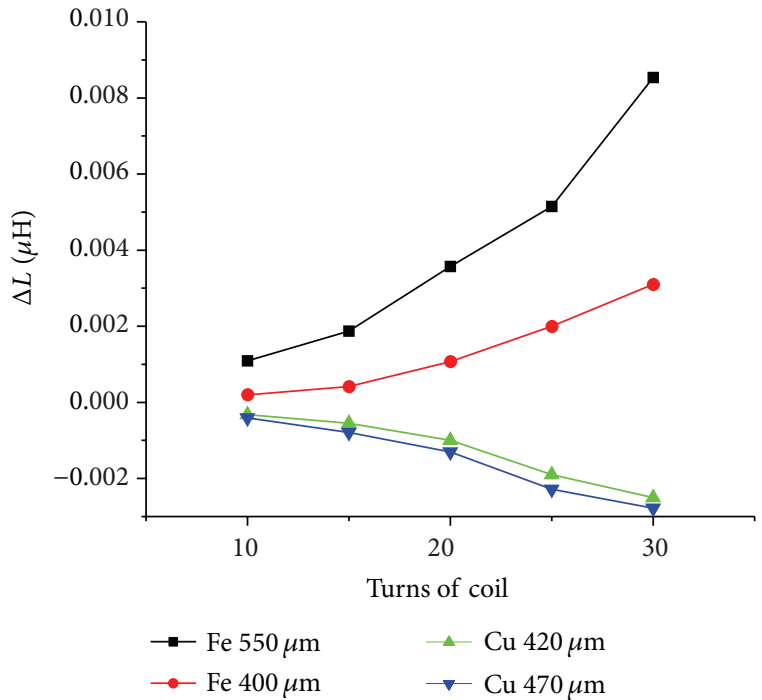

(b)

Figure 9: Inductance changes for the different planar coils by 4 different particles under $2 \mathrm{MHz}$ excitation. (a) Numerical simulations. (b) Experimental results $\left(\mathrm{C}_{5}, \mathrm{C}_{6}, \mathrm{C}_{7}, \mathrm{C}_{8}\right.$, and $\left.\mathrm{C}_{9}\right)$.

slope with particle diameter increase. That means the bigger copper particle causes the larger inductance change. The value is negative because the eddy current decreases the coil inductance [5]. Like iron particle test, the more turns of coil also contribute to the larger inductance change.

Figure 8 shows how the excitation frequency influences the inductance change for the coil $\mathrm{C}_{1}$ (35 turns). For the iron particles, the inductance change decreased slightly as frequency increased, and the curve is a flat slope in the chart, while for copper particles the inductance changes decrease significantly as frequency increases, and the curve is steeper. During the experiment, the particles reciprocate inside the microchannel, which are driven by a syringe pump. At the same time the excitation frequency is adjusted manually.

Figure 9 shows the inductance changes for different planar coils by 4 particles (Fe $(550 \mu \mathrm{m}), \mathrm{Fe}(400 \mu \mathrm{m}), \mathrm{Cu}$ $(420 \mu \mathrm{m})$, and $\mathrm{Cu}(470 \mu \mathrm{m}))$. The coils are of the same outer diameter $(10.64 \mathrm{~mm})$. In the experiment, 5 planar coils are 
used $\left(\mathrm{C}_{5}, \mathrm{C}_{6}, \mathrm{C}_{7}, \mathrm{C}_{8}\right.$, and $\left.\mathrm{C}_{9}\right)$. The excitation frequency is $2 \mathrm{MHz}$. It is obvious that a denser planar coil contributes to a bigger inductance change for the same size particle, which means more sensitivity for particle detection. The experimental results coincide well with the numerical simulation, either for ferromagnetic particles or for nonferromagnetic particles.

\section{Conclusion}

This study has presented the measurement mechanism of a microfluidic chip for metal particle detection using the magnetic vector potential model of the particle-planar coil system which is derived from the complex Helmholtz equation.

The numerical simulation shows good agreement with the experimental data, including the gap width, coil density, turns of the coil, and frequency. This shows that the parameters influence the inductance change. The size of particles can also be evaluated by the proposed model.

This model is established from a perfect ball without planar thickness and line width, which shall be studied in the future work.

\section{Conflict of Interests}

The authors declare that there is no conflict of interests regarding the publication of this paper.

\section{Acknowledgments}

Support for this work was provided by the following: the Natural Science Foundation of China (51205034, 51109021), Scientific Research Program of the Education Department of Liaoning Province (L2012179), and Scientific Research Program of the Ministry of Transport (2013329225260).

\section{References}

[1] J. Tucker, T. Galie, A. Schultz et al., "Lasernet fines optical wear debris monitor: a navy shipboard evaluation of CBM enabling technology," in Proceedings of the 54th International Conference of Machinery Failure Prevention Technology (MFPT '00), pp. 191202, 2000.

[2] J. L. Miller and D. Kitaljevich, "In-line oil debris monitor for aircraft engine condition assessment," in Proceedings of the 2000 IEEE Aerospace Conference, pp. 49-56, March 2000.

[3] H. Zhang, C. H. Chon, X. Pan, and D. Li, "Methods for counting particles in microfluidic applications," Microfluidics and Nanofluidics, vol. 7, no. 6, pp. 739-749, 2009.

[4] L. Du and J. Zhe, "Parallel sensing of metallic wear debris in lubricants using undersampling data processing," Tribology International, vol. 53, pp. 28-37, 2012.

[5] L. Du, J. Zhe, J. Carletta, R. Veillette, and F. Choy, "Realtime monitoring of wear debris in lubrication oil using a microfluidic inductive Coulter counting device," Microfluidics and Nanofluidics, vol. 9, no. 6, pp. 1241-1245, 2010.

[6] H. Zhang, W. Huang, Y. Zhang, Y. Shen, and D. Li, "Design of the microfluidic chip of oil detection," Applied Mechanics and Materials, vol. 117-119, pp. 517-520, 2012.
[7] P. G. Huray, Maxwell's Equations, pp. 209-227, John Wiley and Sons, Hoboken, NJ, USA, 2011.

[8] K. Umashankar, Introduction to Engineering Electromagnetic Fields, pp. 305-333, World Scientific, Singapore, 1989.

[9] E. W. Weisstein, CRC Concise Encyclopedia of Mathematics, CRC Press, Boca Raton, Fla, USA, 2nd edition, 2002.

[10] Y. Lei, J. Liu, Y. Xu, and D. Wang, "Analytical solutions to sphere and coil coaxial eddy current problems," Proceedings of the Chinese Society of Electrical Engineering, vol. 19, no. 2, pp. 26-31, 1999.

[11] I. I. Khandaker, E. Glavas, and G. R. Jones, "A fibre-optic oil condition monitor based on chromatic modulation," Measurement Science and Technology, vol. 4, no. 5, pp. 608-613, 1993.

[12] Z. Zhang, D. Yu, and J. Su, "Fluid power systems particle contamination monitoring based on matched filter," Mechanical Engineering, vol. 39, no. 5, pp. 65-70, 2003.

[13] Y. Li, Y. Zheng, and R. Wang, "Theoretical model and experimental research of optoelectronic granular solid flowmeter," Chinese Journal of Mechanical Engineering, vol. 40, no. 8, pp. 160-165, 2004.

[14] J. Edmonds, M. S. Resner, and K. Shkarlet, "Detection of precursor wear debris in lubrication systems," in Proceedings of the 2000 IEEE Aerospace Conference, pp. 73-78, March 2000.

[15] J. Zhang, B. W. Drinkwater, and R. S. Dwyer-Joyce, "Monitoring of lubricant film failure in a ball bearing using ultrasound," Journal of Tribology, vol. 128, no. 3, pp. 612-618, 2006.

[16] M. Li, K. Zhao, Y. Song et al., "Microfluidic capacitance sensor for detecting metal wear debris in lubrication oil," Journal of Dalian Maritime University, no. 3, pp. 42-46, 2013.

[17] S. Raadnui and S. Kleesuwan, "Low-cost condition monitoring sensor for used oil analysis," Wear, vol. 259, no. 7, pp. 1502-1506, 2005.

[18] J. Davis, J. Carletta, R. Veillette et al., "Instrumentation circuitry for an inductive wear debris sensor," in Proceedings of the IEEE 10th International New Circuits and Systems Conference (NEWCAS '12), pp. 501-504, 2012. 

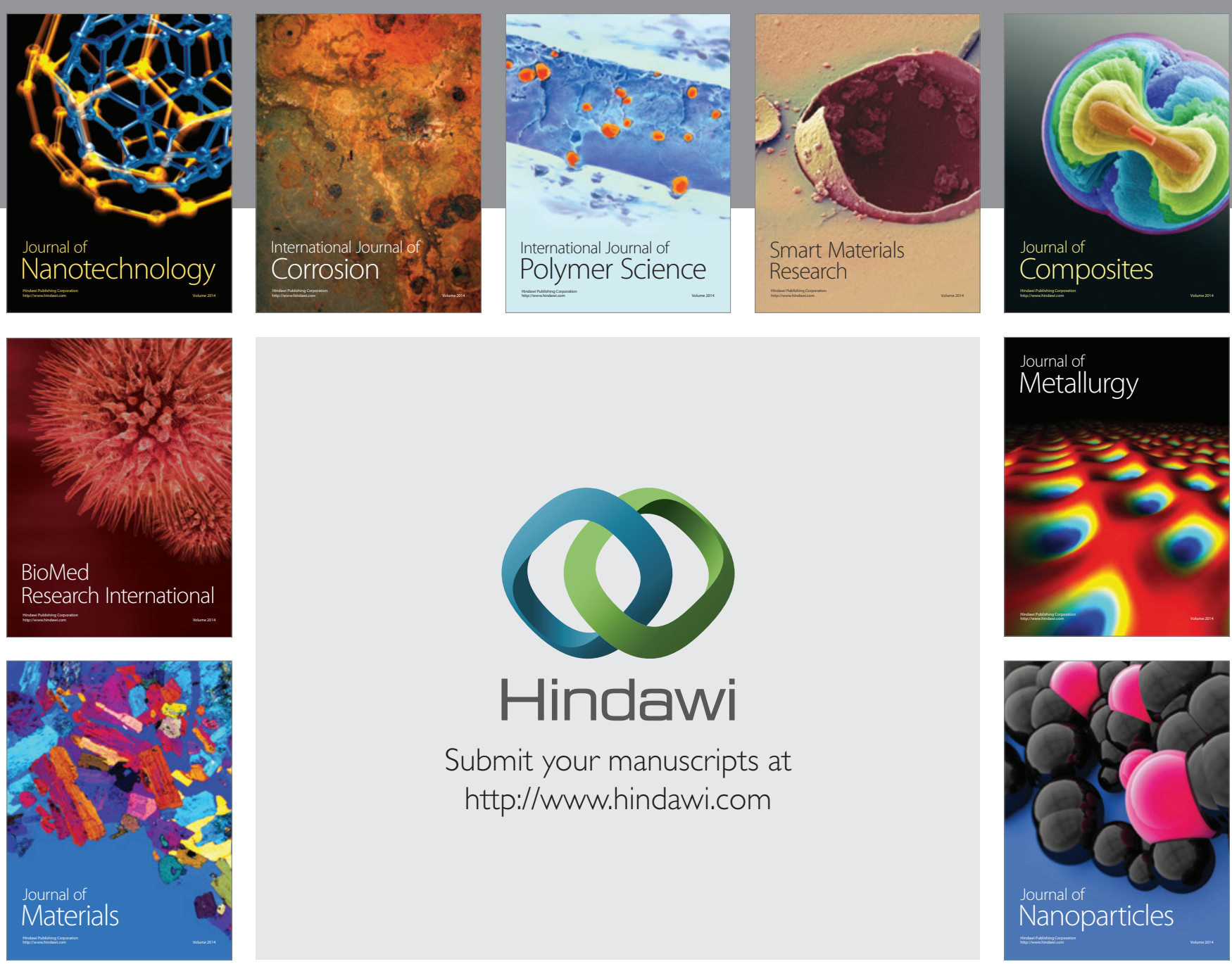

Submit your manuscripts at http://www.hindawi.com
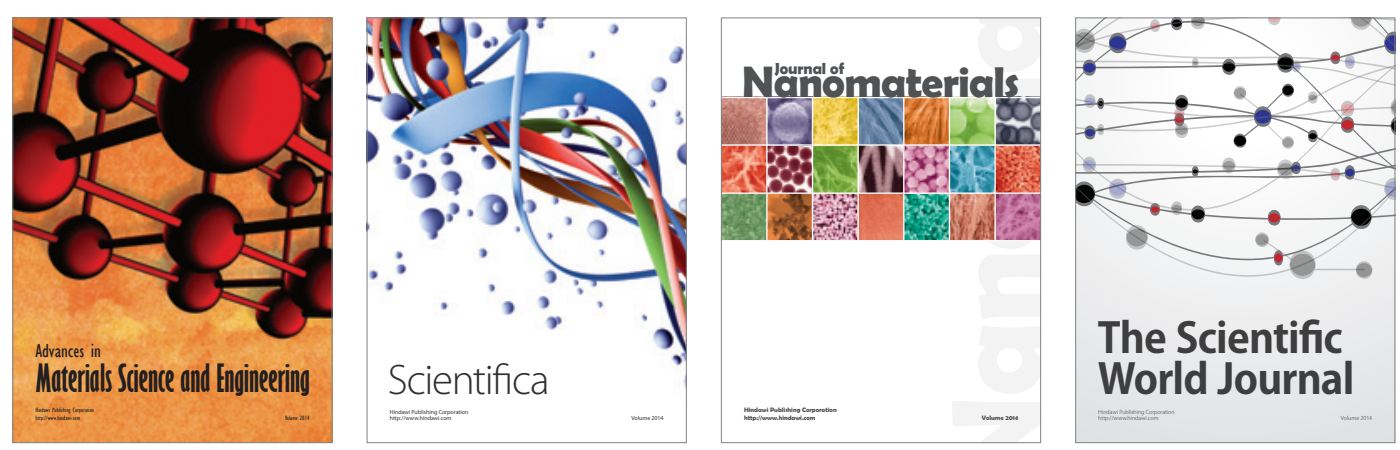

\section{The Scientific World Journal}
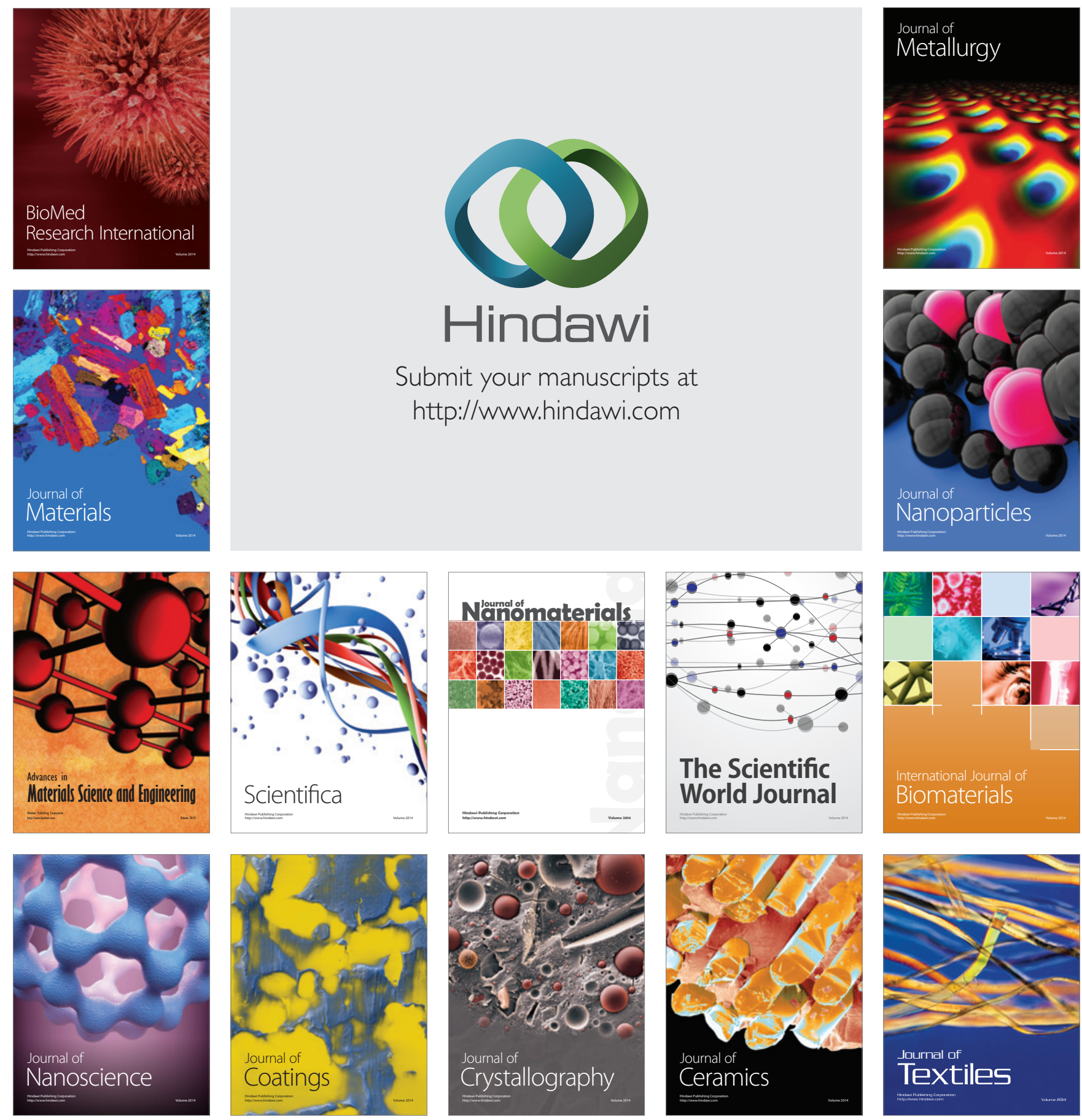